\title{
Método para aplicação de gráficos de controle de regressão no monitoramento de processos
}

\author{
Danilo Cuzzuol Pedrinia,*, Carla Schwengber ten Caten ${ }^{b}$ \\ a,*danilo@producao.ufrgs.br, UFRGS, Brasil \\ btencaten@producao.ufrgs.br, UFRGS, Brasil
}

\begin{abstract}
Resumo
Este artigo propõe um método para a aplicação do gráfico de controle de regressão no monitoramento de processos industriais. Visando facilitar a aplicação do gráfico, o método é apresentado em duas fases: análise retrospectiva (Fase 1) e monitoramento do processo (Fase 11), além de incluir uma modificação do gráfico de controle de regressão múltipla, permitindo o monitoramento direto da característica de qualidade do processo ao invés do monitoramento dos resíduos padronizados do modelo. Também é proposto o gráfico de controle de extrapolação, que verifica se as variáveis de controle extrapolam o conjunto de valores utilizado para estimar o modelo de regressão. 0 método foi aplicado em um processo de uma indústria de borrachas. 0 desempenho do gráfico de controle foi avaliado pelo Número Médio de Amostras (NMA) até o sinal através do método de Monte Carlo, mostrando a eficiência do gráfico em detectar algumas modificações nos parâmetros do processo.
\end{abstract}

Palavras-chave

Gráfico de controle de regressão. Modelos de regressão linear. Número médio de amostras.

\section{Introdução}

Os gráficos de controle de Shewhart destacam-se dentre as ferramentas do Controle Estatístico de Processos (CEP) devido à sua simplicidade operacional e efetividade na detecção de problemas no processo, sendo utilizados com sucesso no monitoramento do desempenho de muitos processos industriais. Woodall e Montgomery (1999) ressaltam que a utilização dos gráficos de controle clássicos supõe que os dados da característica de qualidade do processo sejam independentes e identicamente distribuídos (iid). De acordo com Aizenchtadt, Ingman e Friedler (2008), se essas suposições não forem verdadeiras, o desempenho do gráfico de controle pode ser insatisfatório, gerando elevado número de alarmes falsos ou perda de eficiência na detecção de causas especiais.

Usualmente, o processo precisa ser controlado pelos operadores através da manipulação de algumas variáveis, definidas como variáveis de controle no presente trabalho. Quando essas variáveis de controle são frequentemente alteradas, viola-se a suposição de iid, podendo modificar a média e o desvio-padrão da característica de qualidade. Nesse caso, uma possível solução seria o uso de um gráfico de controle para cada ajuste, mas isso pode ser inviável devido ao baixo número de amostras disponíveis em cada ajuste das variáveis de controle e ao grande número de trocas de gráficos que seria necessário. Outro problema decorrente dessa situação acontece quando alguma variável de controle do processo é propositalmente alterada para um valor extremo, fazendo com que a característica de qualidade assuma um valor muito discrepante. Nessas duas situações, os gráficos de controle clássicos indicariam que o processo está fora de controle, embora aparentemente nada de errado tenha ocorrido com o processo (SHU; TSUNG; TSUI, 2004).

Em ambos os casos, segundo Jacobi, Souza e Pereira (2002) e Shu, Tsung e Tsui (2004), a característica de qualidade de um produto ou processo é mais bem representada pelo seu relacionamento com as variáveis de controle. Uma 
forma de monitorar um processo desse tipo é através dos chamados gráficos de controle baseados em modelos de regressão (LOREDO; JEARKPAPORN; BORROR, 2002). A origem dessa linha de pesquisa é atribuída a Mandel (1969), que propôs o gráfico de controle de regressão. Ele controla a variação média da característica de qualidade, que ocorre em função da alteração do ajuste das variáveis de controle do processo, ao invés de controlar uma média constante do processo, como é usualmente feito nos gráficos de controle de Shewhart. Woodall e Montgomery (1999) e Woodall (2000) apontam os gráficos de controle baseados em modelos de regressão como sendo uma das técnicas desenvolvidas na teoria com grande potencial de aplicação prática.

A proposta original de Mandel (1969) somente pode ser aplicada em processos que apresentem uma variável de controle. Haworth (1996) estendeu a ideia de Mandel (1969) com a proposta do gráfico de controle de regressão múltipla, que consiste na estimativa de um modelo de regressão linear múltipla e no posterior monitoramento dos resíduos padronizados. Embora tenha apresentado uma modificação bastante útil, Haworth (1996) não apresenta um método suficientemente claro para a aplicação do gráfico proposto.

Dessa forma, o presente artigo tem como objetivo propor um método simples para aplicação do gráfico de controle de regressão no monitoramento de processos produtivos com uma ou mais variáveis de controle. 0 método proposto está estruturado em duas fases: Fase 1 (análise retrospectiva) e Fase 11 (monitoramento do processo). 0 método também contempla uma simples modificação ao gráfico de controle de regressão múltipla, fazendo com que seja possível monitorar o valor observado da característica de qualidade, ao invés do monitoramento dos resíduos do modelo de regressão, facilitando a interpretação por parte dos operadores. Adicionalmente, propõe-se o gráfico de controle de extrapolação, que deve ser utilizado conjuntamente com o gráfico de controle de regressão para verificar se o ajuste da variável de controle não extrapola o intervalo de investigação utilizado na modelagem da equação de regressão.

Ao final do trabalho são apresentados os resultados do número médio de amostras (NMA) do gráfico de controle de regressão proposto, de forma a caracterizar o desempenho deste quando ocorrem mudanças pré-estabelecidas nos parâmetros.

\section{Modelos de regressão linear múltipla}

De acordo com Montgomery, Vining e Peck (2001), o modelo de regressão linear múltipla que relaciona a característica de qualidade $y$ às $k$ variáveis de controle do processo é representado na Equação 1. $y=x \beta+\varepsilon$

onde:

$$
y=\left[\begin{array}{c}
y_{1} \\
y_{1} \\
\vdots \\
y_{n}
\end{array}\right] \quad X=\left[\begin{array}{cccc}
1 & x_{11} & \cdots & x_{1 k} \\
1 & x_{21} & \cdots & x_{2 k} \\
\vdots & \vdots & & \vdots \\
1 & x_{n 1} & \cdots & x_{n k}
\end{array}\right] \quad \beta=\left[\begin{array}{c}
\beta_{0} \\
\beta_{1} \\
\vdots \\
\beta_{k}
\end{array}\right] \quad \varepsilon=\left[\begin{array}{c}
\varepsilon_{0} \\
\varepsilon_{1} \\
\vdots \\
\varepsilon_{n}
\end{array}\right]
$$

Na Equação 1, yé o vetor dos valores observados da característica de qualidade, $X$ é a matriz dos valores das $n$ observações das $k$ variáveis de controle, $\beta$ é o vetor de coeficientes de regressão e $\varepsilon$ é o vetor dos erros aleatórios. 0 vetor $\varepsilon$ é estimado pelo vetor de resíduos $e$, definido como a diferença entre os valores observados e os valores estimados pelo modelo $(\hat{y})$, como mostrado na Equação 2.

$e=y-\hat{y}$

Segundo Montgomery, Vining e Peck (2001) e Neter et al. (2005), se o número de observações (n) for maior que o número de variáveis de controle, para estimar a equação de regressão e, por consequência, estimar $y$, utiliza-se o método de Mínimos Quadrados Ordinários (MQO), que visa minimizar as somas quadráticas dos resíduos $e_{i}$. 0 estimador de MQO de $\beta$ é apresentado na Equação 3.

$\widehat{\beta}=\left(X^{\prime} X\right)^{-1} X^{\prime} y$

De acordo com Montgomery, Vining e Peck (2001), para utilizar o MQ0 é necessário assumir que os resíduos sejam independentemente distribuídos, com média igual a 0 e desvio-padrão constante. Ressalta-se que, para realizar inferências estatísticas para os coeficientes de regressão e estimativas da característica da qualidade, é necessário assumir que os resíduos sejam normalmente distribuídos. Ambas as suposições devem ser verificadas após a estimação do modelo, para validação do mesmo. A estimativa da variância dos resíduos do modelo de regressão é dada pelo quadrado médio dos resíduos (QMR):

$$
\hat{\sigma}^{2}=\frac{e^{\prime} e}{n-p}=\frac{S Q R}{n-p}=Q M R
$$

onde: $p=k+1$

De acordo com Neter et al. (2005), a aplicação mais comum dos modelos de regressão é estimar a característica de qualidade $y_{i}$ para um vetor $x_{i}$ de variáveis de controle. Existem duas possibilidades: (i) estimação da resposta média; e (ii) predição de novas observações individuais. A diferença entre essas duas aplicações recai no fato de que a estimativa da resposta média é calculada com base em um vetor $x_{i}$ que foi utilizado para estimar o 
modelo de regressão, enquanto a previsão de novos valores é a estimativa através de um novo vetor $x_{j}$ de variáveis de controle. Em ambos os casos, a estimativa da característica de qualidade é obtida com o uso da estimativa da Equação 5 e do vetor de variáveis de controle $x_{i}$ :

$\hat{y}_{i}=x_{i} \hat{\beta}$

Essas estimativas apresentam diferentes variabilidades, sendo que para a estimativa da resposta média a variância é calculada pela Equação 6.

$\operatorname{var}\left(\mu_{y / x_{i}}\right)=\operatorname{var}(x \beta)=\bar{\sigma}^{2} x\left(X^{\prime} X\right)^{-1} x_{i}$

Para a predição de uma nova observação, é necessário assumir que o modelo de regressão estimado seja válido para o novo conjunto de dados e que essa observação seja independente dos dados utilizados para a estimação do modelo de regressão. Segundo Montgomery et al. (2001), esta independência faz com que a variância de uma nova observação seja maior que a estimativa de resposta média, conforme Equação 7.

$\operatorname{Var}\left(\hat{y}_{j}\right)=\widehat{\sigma}^{2}\left(1+x_{j}^{\prime}\left(X^{\prime} X\right)^{-1} x_{j}\right)$

\section{Gráfico de controle de regressão}

Em linhas gerais, o gráfico de controle de regressão consiste no ajuste de um modelo de regressão linear simples que relacione a característica de qualidade do processo a uma variável de controle e o monitoramento dos valores observados, em comparação com valores ajustados pelo modelo (MANDEL, 1969; JACOBI; SOUZA; PEREIRA, 2002).

No gráfico de controle de regressão de Mandel (1969), a linha central e os limites de controle serão inclinados em relação ao eixo horizontal do gráfico, sendo estimada pela equação de regressão. Os limites do gráfico de controle de regressão são paralelos à linha central, sendo posicionados desta a $L$ desvios-padrão, que é obtido pela raiz quadrada do QMR.

$$
\begin{aligned}
& L S C_{i}=\hat{y}_{i}+L \sqrt{Q M R} \\
& L C_{i}=\hat{y}_{i} \\
& L I C_{i}=\hat{y}_{i}-L \sqrt{Q M R}
\end{aligned}
$$

$\mathrm{Na}$ maioria dos trabalhos consultados, o valor da constante $L$ é 2 ou 3, conforme a sensibilidade e número de alarmes falsos desejados para o gráfico de controle. Mandel (1969) adotou dois desviospadrão como critério.
No gráfico de controle de regressão de Mandel (1969), o eixo das abscissas deixa de ser o número da amostra, passando a ser o valor das variáveis de controle. Com isso, perde-se a ordem temporal dos dados, o que dificulta a realização e interpretação gráfica dos demais testes de estabilidade dos gráficos de controle apresentados em Montgomery (2004).

Outra desvantagem da proposta de Mandel é a restrição de ser utilizada apenas em processos com uma variável de controle. Como solução a esta restrição, Haworth (1996) propôs o gráfico de controle de regressão múltipla, que utiliza os resíduos padronizados na forma Student como variável monitorada ao longo do tempo. De acordo com Haworth (1996) e Shu, Tsung e Tsui (2004), esses resíduos são obtidos pela Equação 12.

$$
z_{j}=\frac{e_{j}}{\bar{\sigma} \sqrt{1+x_{j}^{\prime}\left(X^{\prime} X\right)^{-1} x_{j}}}
$$

A linha central do gráfico de controle de regressão múltipla é igual a zero, que é a média dos resíduos de um modelo de regressão. Os limites de controle são baseados no valor da estatística t-Student, com nível de confiança $1-\alpha$ e $n-p$ graus de liberdade.

$$
\begin{aligned}
& L S C=+t_{\alpha / 2, n-p} \\
& L C=0 \\
& L I C=-t_{\alpha / 2, n-p}
\end{aligned}
$$

Uma falha do procedimento apresentado por Haworth (1996) é não apresentar os limites de controle para a Fase 1 de execução do gráfico de controle de regressão múltipla, já que os resíduos na forma Student são obtidos pelo uso do desviopadrão de uma nova observação.

Loredo, Jearkpaporn e Borror (2002) e Shu, Tsung e Tsui (2004) também propuseram alternativas que permitem o uso de modelos de regressão linear múltipla. No primeiro trabalho, utiliza-se a amplitude móvel dos resíduos para estimar o desvio-padrão do gráfico de controle de regressão. Shu, Tsung e Tsui (2004) apresentam o gráfico EWMAREG, que consiste no monitoramento dos resíduos padronizados através de um gráfico de controle de médias móveis exponencialmente ponderadas (EWMA).

As propostas de Haworth (1996), Loredo, Jearkpaporn e Borror (2002) e Shu, Tsung e Tsui (2004) apresentam a vantagem de manter a ordem temporal dos dados, que não era possível na proposta inicial de Mandel (1969). Exemplos de aplicações 
do gráfico de controle de regressão podem ser encontrados em Johnson, B., Johnson, C. e Seiber (1995), Olin (1998), Jacobi, Souza e Pereira (2002), Omura e Steffe (2003) e Aizenchtadt, Ingman e Friedler (2008).

Também são encontrados na literatura diversos procedimentos similares aos gráficos de controle de regressão, procedimentos estes que podem ser incluídos na linha de pesquisa dos gráficos de controle baseados em modelos. Por exemplo, Zhang (1985) propôs o gráfico de controle para seleção de causas (GCSC), que são aplicados no monitoramento de processos compostos por várias atividades sequenciais, também chamados de processos em cascata. Wade e Woodall (1993) revisaram o GCSC e propuseram limites de controles a partir da previsão de novas observações, mostrando que estes podem ser mais eficientes que a proposta inicial de Zhang (1985).

Hawkins (1991) propôs um procedimento para o monitoramento de processos multivariados baseado em variáveis ajustadas por regressão. Posteriormente, Hawkins (1993) adaptou essa técnica para processos em cascata, como uma alternativa ao GCSC. Hauck, Runer e Montgomery (1999) utilizaram regressões agrupadas para o caso de processos em cascata multivariados.

Olin (1998) apresentou o gráfico de controle de regressão não linear, além de propor o uso de modelos lineares generalizados (MLG) para o monitoramento de processos. Skinner, Montgomery e Runger (2003) e Jearkpaporn et al. (2003) propuseram o uso de gráficos de controle para resíduos deviance dos MLGs no monitoramento de contagem de não conformidades com distribuição Poisson e Gama, respectivamente. Posteriormente, Jearkpaporn et al. (2007) estenderam esse método para processos em cascata, de maneira similar ao GCSC.

Kang e Albin (2000) propuseram um procedimento para o monitoramento de perfis lineares, onde é requerido o ajuste de modelos de regressão linear simples para cada amostra para verificar alterações nos parâmetros dos perfis, como os coeficientes de regressão e variabilidade do processo. Para maiores detalhes sobre o monitoramento de perfis, Woodall et al. (2004) e Woodall (2007) expõem revisões sobre os avanços nessa área.

Loredo, Jearkpaporn e Borror (2002) e Montgomery (2004) também destacam a aplicação do gráfico de controle de regressão para processos autocorrelacionados, em substituição aos gráficos de controle baseados em modelos para séries temporais, se os valores das variáveis de controle forem conhecidos. Segundo esses autores, os resíduos de um modelo de regressão usualmente não apresentam autocorrelação, mesmo que a característica de qualidade ou as variáveis de controle sejam autocorrelacionadas. Montgomery (2004) também cita a possibilidade de adaptação do gráfico de controle de regressão para o monitoramento de processos que apresentam tendências, que podem ser causadas, por exemplo, pelo desgaste de ferramentas.

\section{Método proposto}

Para Woodall e Montgomery (1999) e Vining (2009), um método de implantação de gráficos de controle pode ser dividido em duas fases: (i) Fase 1, chamada de análise retrospectiva, que é utilizada quando os parâmetros do processo são desconhecidos e devem ser estimados com base em uma amostra proveniente do processo a ser monitorado; (ii) Fase 1l, ou monitoramento do processo, que é utilizada quando os parâmetros do processo são previamente conhecidos, ou foram estimados na Fase 1. De acordo com Faltin et al. (1997), a Fase 11 requer a suposição de que os dados da característica de qualidade monitorados e os dados utilizados na estimação dos limites de controle da Fase 1 sigam a mesma distribuição de probabilidade.

Essa divisão em duas fases é bastante útil na aplicação dos gráficos de controle, especialmente nos procedimentos mais complexos desenvolvidos no meio acadêmico, já que apresenta ao leitor todos os procedimentos, desde a estimação de parâmetros até a identificação de causas especiais. Segundo Faltin et al. (1997), a maioria dos trabalhos encontrados na literatura sobre gráficos de controle falha justamente em não separar essas duas fases em sua metodologia, o que dificulta a disseminação e aplicação dessas novas técnicas no meio industrial. lsso também foi verificado nos trabalhos sobre gráfico de controle de regressão consultados durante o levantamento bibliográfico para o presente artigo. Como será apresentado posteriormente, os limites de controle do gráfico de controle de regressão para as Fases 1 e 11 são diferentes, logo a apresentação do presente método em duas fases visa auxiliar a aplicação correta dos limites em cada fase.

0 método proposto contempla uma modificação relativamente simples que permitirá o monitoramento direto das observações referentes a uma característica de qualidade dependente de uma ou mais variáveis de controle do processo, ao invés do monitoramento dos resíduos da regressão. A modificação proposta no presente trabalho é 
similar à modificação do gráfico de controle EWMA apresentada por Montgomery e Mastrangelo (1991) e será apresentada no Apêndice 1.

Montgomery e Mastrangelo (1991) e Montgomery (2004) ressaltam que alguns operários têm dificuldades em interpretar os gráficos de controle baseados em resíduos, já que estes nem sempre são considerados pelos operários como sendo uma referência direta do processo. Esses autores recomendam a utilização de procedimentos que monitorem diretamente as características de qualidade, em substituição ao monitoramento de resíduos, já que este tipo de modificação também combina informações sobre a dinâmica do processo e o controle estatístico em um único gráfico.

Antes da descrição do método proposto é necessário apresentar as três suposições necessárias à aplicação deste: (i) a característica de qualidade do processo deve ser uma variável contínua e sua relação com as variáveis de controle deve ser ajustável por um modelo de regressão linear; (ii) os resíduos da regressão devem ser normal $\mathrm{e}$ independentemente distribuídos, com média zero e desvio-padrão constante; e (iii) seguindo Faltin et al. (1997), assume-se que os dados utilizados para o ajuste da regressão na Fase 1 do método e os dados monitorados na Fase 11 devem ter o mesmo comportamento.

\subsection{Fase 1 - análise retrospectiva}

As etapas de execução desta fase são: (i) coleta de dados; (ii) ajuste do modelo de regressão linear simples ou múltipla; e (iii) análise de estabilidade da Fase 1, que é a detecção de causas especiais.

\subsubsection{Coleta de dados}

Para a Fase 1 do método proposto é necessário coletar uma amostra significativa do processo, que permita estimar todos os coeficientes de regressão, além de disponibilizar o maior número possível de graus de liberdade para a estimativa do termo de erro do modelo, aumentando a confiabilidade dos resultados. Os dados coletados devem conter o valor da característica de qualidade monitorada e os valores das variáveis de controle do processo referentes ao ajuste do processo no momento de coleta. Os dados utilizados no ajuste do modelo de regressão podem ser históricos ou oriundos de uma coleta através de um planejamento experimental.

\subsubsection{Estimação do modelo de regressão}

De posse dos dados, utiliza-se o MQO para estimar o modelo de regressão linear que relacione a característica de qualidade do processo às variáveis de controle. Após a estimativa do modelo de regressão, realizam-se seis passos para a aceitação do modelo: (i) teste de significância do modelo de regressão; (ii) uso do fator de inflação da variância (FIV) para a verificação da presença de multicolinearidade no modelo; (iii) teste dos coeficientes individuais de regressão, de forma a verificar a significância da relação entre cada uma das variáveis de controle e a característica de qualidade; (iv) uso da distância de Cook para análise de pontos influentes na estimativa do modelo; (v) análise das suposições de que os resíduos são normalmente distribuídos com média zero e desvio-padrão aproximadamente constante; e (vi) uso da estatística de Durbin-Watson para verificar a hipótese de que os resíduos são independentes. Ressalta-se que os passos (v) e (vi) visam validar o modelo de regressão e as inferências estatísticas realizadas. Para maiores detalhes sobre esses passos, sugere-se Montgomery, Vining e Peck (2001) e Neter et al. (2005).

\subsubsection{Análise de estabilidade da Fase 1}

Ressalta-se que para o gráfico de controle de regressão, o valor de referência para a característica de qualidade, dado o ajuste das variáveis de controle do processo, é obtido pelo valor previsto pelo modelo de regressão. Para a Fase 1 do método proposto, utilizam-se os limites de controle do gráfico de regressão apresentados anteriormente, adotando-se $L=3$. Dessa forma, os limites de controle da Fase 1 são:

$L S C_{i}=\hat{y}_{i}+3 \sqrt{\hat{\sigma}^{2}}=\hat{y}_{i}+3 \sqrt{Q M R}$

$L C_{i}=\hat{y}_{i}$

$L I C_{i}=\hat{y}_{i}-3 \sqrt{\hat{\sigma}^{2}}=\hat{y}_{i}-3 \sqrt{Q M R}$

Esses limites de controle são semelhantes aos adotados por Mandel (1969), porém nesse caso as amostras são plotadas no gráfico em ordem temporal, ao invés de serem plotadas de acordo com o valor da variável de controle, permitindo a adoção de todos os oito testes de estabilidade descritos por Montgomery (2004). Assim, os limites de controle e a linha central serão linhas móveis, variando de acordo com o valor previsto pelo modelo de regressão. Em relação ao gráfico de controle de regressão múltipla de Haworth (1996), monitora-se o valor observado da característica de qualidade, ao invés do monitoramento dos resíduos padronizados.

Para o método proposto, será considerado apenas o teste de um ponto excedendo os limites de controle. Se forem detectadas causas especiais, a equação de regressão e os limites de controle deverão 
ser calculados novamente, desconsiderando-se os pontos fora de controle. Caso não existam novos pontos fora de controle, procede-se para a Fase 11 do método proposto. Esse procedimento é similar à análise de outliers encontrada na maioria dos textos sobre modelos de regressão, que é usualmente empregada após a estimativa de um modelo.

\subsection{Fase ll - monitoramento do processo}

Para essa fase, assume-se que o modelo de regressão estimado na Fase 1 do método proposto está correto e que os dados do processo a ser monitorado apresentem o mesmo comportamento dos dados utilizados na Fase 1. Assim, é possível usar a equação de regressão e o desvio-padrão do modelo para calcular o valor previsto da característica de qualidade. As etapas de execução da Fase 11 são: coleta de dados e monitoramento do processo propriamente dito.

\subsubsection{Coleta de dados}

Para a Fase 11 do método proposto é necessária a coleta de amostras individuais do processo, obtidas em intervalos regulares de tempo. Ressalta-se que os dados coletados devem conter o valor da característica de qualidade monitorada e respectivos valores das variáveis de controle do processo.

\subsubsection{Monitoramento do processo propriamente dito}

Primeiramente, é necessário monitorar as variáveis de controle da amostra coletada, com a finalidade de verificar se as amostras extrapolam a região original definida pelo conjunto de variáveis de controle utilizadas para estimar o modelo de regressão. Essa verificação é necessária, já que, de acordo com Montgomery, Vining e Peck (2001) e Neter et al. (2005), o modelo de regressão é uma boa aproximação para a relação entre a característica de qualidade e as variáveis de controle dentro do intervalo de investigação, mas pode ser uma estimativa muito pobre para valores das variáveis de controle fora desse intervalo, prejudicando o desempenho do gráfico de controle de regressão múltipla.

Para essa verificação, adaptou-se graficamente o procedimento descrito por Montgomery, Vining e Peck (2001), dando origem ao gráfico de controle de extrapolação. Nele, a variável a ser monitorada é o $h_{j j}$, apresentada na Equação 19.

$h_{j j}=x_{j}^{\prime}\left(X^{\prime} X\right)^{-1} x_{j}$

onde: $x_{j}$ é o vetor das variáveis de controle da j-ésima nova observação.
0 gráfico de controle de extrapolação apresenta a particularidade de possuir apenas o LSC, que é o valor máximo dos elementos $h_{i i}$ utilizados para estimar o modelo de regressão:

$L S C=\max \left(h_{i i}\right)$

A escolha desse limite de controle é oriunda do procedimento descrito por Montgomery, Vining e Peck (2001), já que o valor máximo de $h_{i i}$ dos dados utilizados para estimar o modelo de regressão delimita a região composta por todos os vetores $x_{i}$. Assim, um ponto que exceda o LSC está fora dessa região, ocorrendo, necessariamente, a extrapolação do modelo de regressão. Especificamente para o gráfico de controle de extrapolação, um ponto fora de controle pode indicar: (i) erro de ajuste do processo por parte dos operadores; (ii) erro de coleta de dados; ou (iii) alteração nos parâmetros de ajuste do processo, indicando a necessidade de reinício da Fase 1 do método proposto. Caso não existam pontos fora de controle no gráfico de controle de extrapolação, prossegue-se para o monitoramento da característica de qualidade.

Para esse monitoramento, propõe-se uma modificação ao gráfico de controle de regressão múltipla, proposto por Haworth (1996). Esta modificação é apresentada no Apêndice 1. Os limites de controle e a linha central dessa modificação são dados pelas Equações 20, 21 e 22.

$$
\begin{aligned}
& L S C_{j}=\hat{y}_{j}+3 \sqrt{\operatorname{QMR}\left(1+h_{j j}\right)} \\
& L C_{j}=\hat{y}_{j} \\
& L I C_{j}=\hat{y}_{j}-3 \sqrt{\operatorname{QMR}\left(1+h_{j j}\right)}
\end{aligned}
$$

0 elemento $h_{j j}$ é utilizado como um fator de correção do desvio-padrão da predição de uma nova observação, já que mede o distanciamento do vetor de variáveis de controle em relação ao vetor composto pelo valor médio de cada variável de controle. Esse fator de correção é o mesmo utilizado para a estimativa de um intervalo de confiança para a previsão de uma nova observação.

Caso uma amostra exceda os limites de controle, o processo será considerado como estando fora de controle estatístico e, dessa forma, as causas especiais que alteraram o estado do processo deverão ser investigadas. Através da experiência prática dos autores, as principais causas de pontos fora de controle no gráfico de controle de regressão múltipla podem ser: (i) ação significativa de um fator não controlável no processo; (ii) existência de uma variável de controle significativa que não foi incluída no modelo; (iii) alteração no valor de 
algum coeficiente de regressão; e (iv) alterações no desvio-padrão dos resíduos.

Para este trabalho, não serão descritas as investigações necessárias para a descoberta e solução das causas especiais que agiram no processo. A indicação, por parte do gráfico de controle de regressão, de muitos pontos fora de controle, pode ser sinal de uma alteração sistemática na relação entre a característica de qualidade e as variáveis de controle do processo, violando-se a suposição (iv) do método proposto. Nesse caso, é necessário realizar a Fase 1 do método proposto novamente, a fim de revisar o modelo de regressão linear múltipla estimado.

\section{Aplicação do método proposto}

0 método proposto foi aplicado em um processo de extrusão de bandas de rodagem de uma indústria multinacional de borrachas. A característica de qualidade a ser monitorada no processo estudado é o tempo de estabilização, medido a partir do momento em que o processo começa a operar após setup de um novo ajuste das variáveis de controle até o momento em que o processo se estabiliza. Essa característica de qualidade é uma variável contínua, que está diretamente relacionada à perda de matéria-prima, já que enquanto o processo está desestabilizado o produto produzido apresenta baixa qualidade, sendo posteriormente descartado.

As variáveis de controle desse processo são: temperatura na fieira $\left(x_{1}\right)$; nivel de plastificação $\left(x_{2}\right)$; parâmetro de carregamento $\left(x_{3}\right)$; fator de enchimento $\left(x_{4}\right)$; temperatura da hélice $\left(x_{5}\right)$; temperatura no eixo Y $\left(x_{6}\right)$ e tempo de $\operatorname{setup}\left(x_{7}\right)$.

\subsection{Aplicação da Fase 1}

Os dados utilizados para a Fase 1 foram obtidos através de um experimento realizado na empresa. Nele, utilizou-se um projeto fatorial fracionado em quatro partes com as variáveis de controle ajustadas em dois níveis $\left(2^{7-2}\right)$, resultando em 32 ensaios. 0 modelo de regressão, estimado pelo método de MQO, é apresentado na Equação 23:

$$
\begin{aligned}
& \hat{y}_{i}=227,1+14,8 x_{1}-16,9 x_{4}+12,9 x_{7}+11,4 x_{1} x_{3} \\
& +14,4 x_{2} x_{5}+11,9 x_{2} x_{6}+8,10 x_{3} x_{4}
\end{aligned}
$$

Esse modelo apresenta um coeficiente de determinação de aproximadamente $76,8 \%$ e um coeficiente de determinação ajustado de $76,1 \%$. Ressalta-se que todas as variáveis de controle inseridas no modelo foram apontadas como estatisticamente significativas nos testes $\mathrm{t}$-individuais e que o teste $\mathrm{F}$ da tabela Anova teve valor $\mathrm{p}$ aproximadamente igual 0 .

Na sequência, utiliza-se o gráfico de controle de regressão da Fase 1 para verificação de estabilidade dos dados utilizados para estimar o modelo de regressão linear. Conforme pode ser observado na Figura 1, não há nenhum ponto fora de controle e o processo pode ser considerado como estando sob controle estatístico. Dessa forma, os parâmetros do modelo de regressão apresentado na Equação 23 podem ser utilizados para monitorar o processo de extrusão de bandas de rodagem.

\subsection{Aplicação da Fase II}

Para a aplicação da Fase 11 do método proposto, foram monitoradas 122 amostras provenientes do processo estudado. Após a coleta dos dados, verifica-se se alguma das amostras coletadas extrapola a região formada pelos dados utilizados para estimar o modelo de regressão na Fase 1. 0 gráfico de controle de extrapolação é apresentado na Figura 2.

Analisando a Figura 2, observa-se que a amostra 41 apresentou um valor de $h_{i j}$ superior ao LSC e, portanto, é considerada como sendo uma amostra que extrapola a região formada pelos dados utilizados para estimar o modelo de regressão.

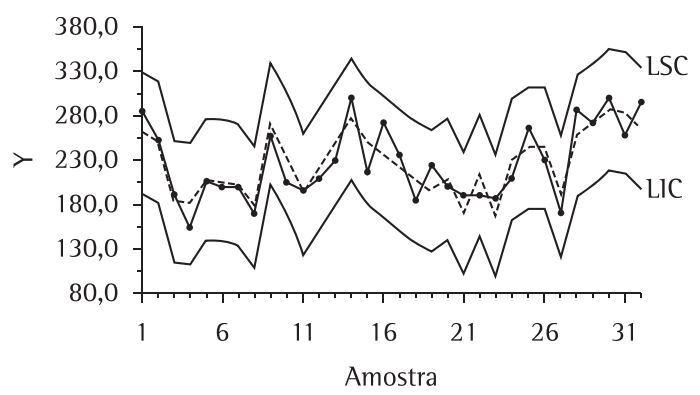

Figura 1. Gráfico de controle de regressão múltipla da Fase 1.

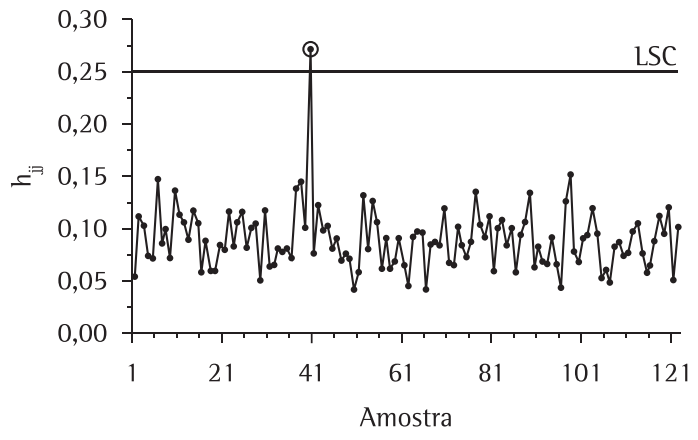

Figura 2. Gráfico de controle de extrapolação. 
Assim, conforme o método proposto, essa amostra será desconsiderada do gráfico de controle de regressão da Fase 11. Após a exclusão da amostra 41, restam 121 amostras que serão monitoradas pelo gráfico de controle de regressão proposto. Adotando o valor do QMR de 488,9, apresentado na Tabela 1, do modelo estimado, os limites de controle do método proposto são:

$$
\begin{aligned}
& L S C_{j}=\hat{y}_{j}+66,3 \sqrt{1+h_{j j}} \\
& L I C_{j}=\hat{y}_{j}-66,3 \sqrt{1+h_{j j}}
\end{aligned}
$$

Na Figura 3 apresenta-se o gráfico de controle de regressão para o monitoramento do processo de linhas de extrusão de bandas de rodagem.

Como é possível observar na Figura 3 , o gráfico de controle de regressão múltipla da Fase 11 proposto indicou duas amostras como estando fora de controle.

\section{Estudos de simulação}

Nesta seção são apresentados os resultados do NMA do método proposto, obtidos através da simulação de Monte Carlo. Os resultados são comparados com a adoção dos limites de controle de Mandel (1969), Haworth (1996) e uma terceira alternativa, em que adotam-se os limites de controle da Fase 1 como limites da Fase 11, identificado como gráfico $3 \sigma$.

É importante destacar que o estudo realizado concentra-se no cálculo do NMA da Fase 11 destes gráficos. Para isso, utiliza-se a Equação 26 como modelo-base a ser simulado:

$y=3+2 x_{1}+x_{2}-4 x_{1} x_{2}+\varepsilon$

onde:

$x_{1} \sim \mathrm{N}(0,1), x_{2} \sim \mathrm{N}(2,1)$ e $\varepsilon \sim \mathrm{N}(0,2)$

$\mathrm{Na}$ simulação da Fase 1, geram-se 50 valores para as variáveis de controle e característica de qualidade, obedecendo à relação descrita pela Equação 26, e estima-se o modelo de regressão com essas amostras. Os parâmetros do modelo são utilizados para a obtenção dos limites de controle da Fase 1 e, se existirem pontos discrepantes, o modelo de regressão é estimado novamente, com a retirada desses pontos fora de controle. Destaca-se que essa retirada de valores discrepantes é realizada apenas uma vez para evitar um "supercontrole" do gráfico, conforme sugerido por Montgomery (2004).

Para simulação da Fase 11 , geram-se amostras seguindo o modelo-base para a estimativa do NMA sob controle $\left(\mathrm{NMA}_{0}\right)$ ou geram-se amostras com alterações nos parâmetros do modelo, para a estimativa do NMA fora de controle $\left(\mathrm{NMA}_{\mathrm{f}}\right)$. As amostras são geradas até que ocorra a primeira amostra fora dos limites de controle do gráfico estudado. Anota-se o número dessa amostra (NA) e reinicia-se a simulação a partir da Fase 1 novamente. O NMA para cada situação foi estimado pela média dos NA.

Foram realizadas alterações nos seguintes parâmetros do modelo-base: coeficientes do modelo de regressão $\left(\beta_{0}, \beta_{1}\right.$ e $\left.\beta_{2}\right)$, desvio-padrão dos resíduos $\left(\sigma_{\mathrm{e}}\right)$ e da variável de controle $x_{1}$. A simulação foi realizada no programa estatístico $\mathrm{R}$ e o número de repetições adotado para cada alteração foi de 10.000, número este que, segundo Kang e Albin (2000), acarreta um erro de menos de 2\%, permitindo boas conclusões acerca do resultado.

$\mathrm{Na}$ análise do NMA, dois aspectos devem ser considerados: (i) o $\mathrm{NMA}_{0}$ deve ser um valor grande, já que representa o número de amostras até a ocorrência de alarmes falsos; e (ii) o $\mathrm{NMA}_{f}$ deve ser um valor pequeno, pois é um indicador da eficiência com que o gráfico de controle detecta as alterações no processo (MONTGOMERY, 2004).

Na Tabela 1 apresenta-se o NMA dos gráficos estudados para alterações no coeficiente de intercepto $\left(\beta_{0}\right)$, em múltiplos do desvio-padrão dos resíduos $\left(\sigma_{\mathrm{e}}\right)$. Segundo Shu, Tsung e Tsui (2004), essas alterações geram perturbações na média dos resíduos. Nota-se que o gráfico de controle de Mandel (1969) apresenta o melhor desempenho na detecção dessas alterações, mas apresenta a desvantagem de ter um baixo $\mathrm{NMA}_{0}$, significando que ocorrerão cerca de 5\% de alarmes falsos.

\begin{tabular}{|c|c|c|c|c|c|c|c|}
\hline \multirow{2}{*}{ Gráfico } & \multirow{2}{*}{$\frac{\mathrm{NMA}_{0}}{\beta_{0}}$} & \multicolumn{6}{|c|}{$\mathrm{NMA}_{\mathrm{f}}$} \\
\hline & & $\beta_{0}+0,5 \sigma_{e}$ & $\beta_{0}+1,0 \sigma_{e}$ & $\beta_{0}+1,5 \sigma_{e}$ & $\beta_{0}+2,0 \sigma_{e}$ & $\beta_{0}+2,5 \sigma_{e}$ & $\beta_{0}+3,0 \sigma_{e}$ \\
\hline Mandel & 20,06 & 13,31 & 6,22 & 3,30 & 2,03 & 1,47 & 1,20 \\
\hline Gráfico $3 \sigma$ & 393,61 & 187,05 & 51,93 & 17,49 & 6,79 & 3,46 & 2,09 \\
\hline Proposta & 653,56 & 300,63 & 75,82 & 22,56 & 8,65 & 4,05 & 2,38 \\
\hline Haworth & 1259,94 & 564,16 & 127,00 & 35,42 & 11,96 & 5,29 & 2,87 \\
\hline
\end{tabular}

Tabela 1. Valores do NMA para alterações no coeficiente de intercepto de $\beta_{0}$. 
$\mathrm{Na}$ análise dos outros gráficos, observa-se que o gráfico de controle de regressão proposto, o gráfico de controle de Haworth (1996) e o gráfico de controle $3 \sigma$ possuem baixas taxas de alarmes falsos. 0 desempenho do gráfico de controle $3 \sigma$ é superior ao dos outros dois, sobretudo para alterações inferiores a $2 \sigma_{\mathrm{e}}$. Para alterações superiores a $2 \sigma_{\mathrm{e}}$, o gráfico de controle proposto e o gráfico $3 \sigma$ apresentam desempenho similar.

$\mathrm{Na}$ Tabela 2 são apresentados os resultados da simulação para alterações no coeficiente de inclinação $\beta_{1}$, também em unidades de $\sigma_{e}$. Como a variável de controle $x_{1}$ possui média 0 , ressalta-se que as alterações em $\beta_{1}$ causarão alterações apenas na variabilidade dos resíduos (SHU; TSUNG; TSUI, 2004). 0 gráfico de Mandel (1969) apresenta um desempenho notoriamente superior aos demais gráficos quando o processo está fora de controle, mas deve-se lembrar novamente que esse gráfico possui um elevado número de alarmes falsos. Para alterações de 1,5 a $3 \sigma_{\mathrm{e}}$, o gráfico de controle proposto e o gráfico $3 \sigma$ apresentam desempenho bastante similar e superior ao gráfico de controle de Haworth (1996).

A terceira simulação estudada foram as alterações no coeficiente de inclinação $\beta_{2}$, em múltiplos de que $\sigma$, cujos resultados são apresentados na Tabela 3. É importante destacar que quando altera-se $\beta_{2}$ essa alterações tanto na média quanto no desvio-padrão dos resíduos, já que a variável de controle $x_{2}$ possui média diferente de zero, equivalendo-se a uma alteração conjunta em $\beta_{0}$ e $\beta_{1}$. Para esta situação, todos os quatro gráficos de controle estudados apresentam desempenhos semelhantes para alterações maiores ou iguais a $1,5 \sigma_{\mathrm{e}} \mathrm{em} \beta_{2}$.

0 NMA dos gráficos estudados, para alterações multiplicativas no desvio-padrão dos resíduos, é apresentado na Tabela 4. 0 desempenho do gráfico de controle de Mandel (1969) é superior aos demais gráficos de controle para todas as simulações de processo fora de controle. 0 gráfico de controle de regressão proposto e o gráfico $3 \sigma$ apresentam desempenhos muito próximos, principalmente para alterações do desvio-padrão dos resíduos superiores a 1,8 .

$\mathrm{Na}$ última situação simulada, apresentada na Tabela 5, alterou-se o valor da variável de controle $x_{1}$. É importante destacar que os gráficos de controle de regressão não devem ser sensiveis a este tipo de alteração, já que as alterações acarretam mudanças de mesma proporção tanto no valor previsto quando real da característica de qualidade.

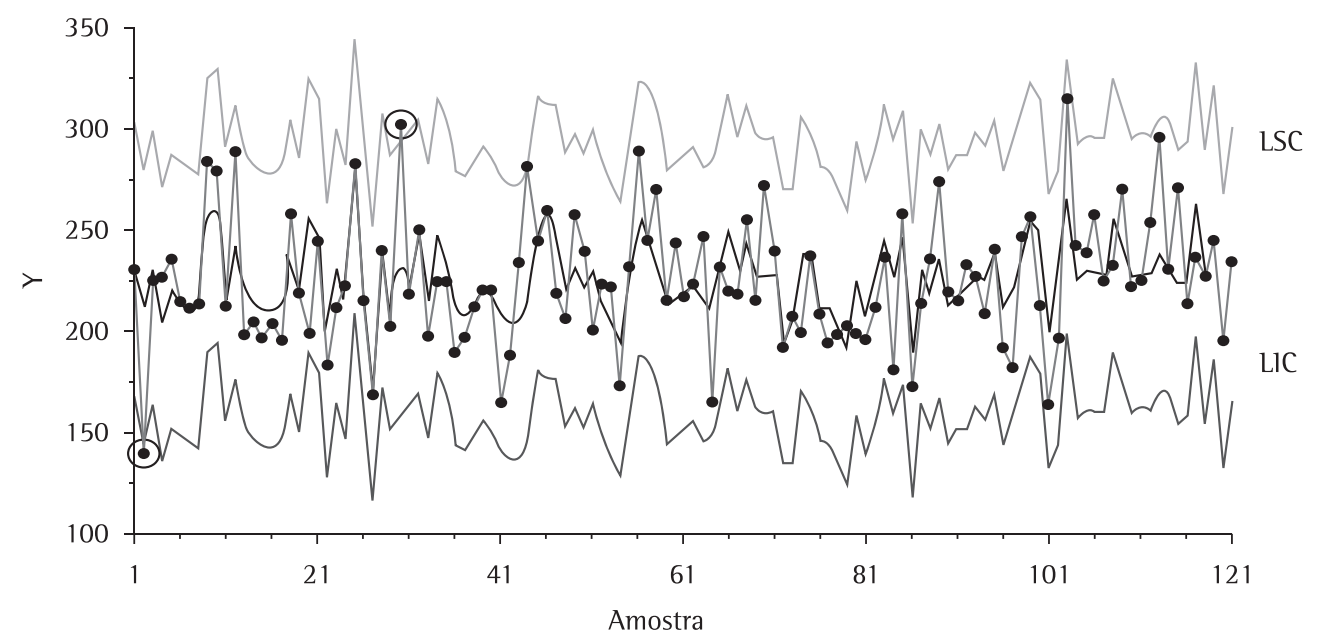

Figura 3. Gráfico de controle de regressão múltipla da Fase 11.

Tabela 2. Valores do NMA para alterações no coeficiente de inclinação de $\beta$.

\begin{tabular}{|c|c|c|c|c|c|c|c|}
\hline \multirow{2}{*}{ Gráfico } & \multirow{2}{*}{$\frac{\mathrm{NMA}_{0}}{\beta_{1}}$} & \multicolumn{6}{|c|}{$\mathrm{NMA}_{f}$} \\
\hline & & $\beta_{1}+0,5 \sigma_{e}$ & $\beta_{1}+1,0 \sigma_{\mathrm{e}}$ & $\beta_{1}+1,5 \sigma_{\mathrm{e}}$ & $\beta_{1}+2,0 \sigma_{\mathrm{e}}$ & $\beta_{1}+2,5 \sigma_{e}$ & $\beta_{1}+3,0 \sigma_{e}$ \\
\hline Mandel & 20,06 & 13,21 & 6,48 & 3,80 & 2,69 & 2,21 & 1,88 \\
\hline Gráfico 3 & 394,82 & 160,16 & 33,66 & 11,04 & 5,80 & 3,81 & 2,92 \\
\hline Proposta & 653,56 & 293,63 & 56,26 & 11,21 & 5,97 & 3,97 & 3,03 \\
\hline Haworth & 1259,94 & 512,66 & 88,11 & 19,53 & 8,24 & 4,79 & 3,47 \\
\hline
\end{tabular}


Tabela 3. Valores do NMA para alterações no coeficiente de inclinação de $\beta$.

\begin{tabular}{|c|c|c|c|c|c|c|c|}
\hline \multirow{2}{*}{ Gráfico } & \multirow{2}{*}{$\frac{\mathrm{NMA}_{0}}{\beta_{2}}$} & \multicolumn{6}{|c|}{$\mathrm{NMA}_{f}$} \\
\hline & & $\beta_{2}+0,5 \sigma_{e}$ & $\beta_{2}+1,0 \sigma_{e}$ & $\beta_{2}+1,5 \sigma_{e}$ & $\beta_{2}+2,0 \sigma_{e}$ & $\beta_{2}+2,5 \sigma_{e}$ & $\beta_{2}+3,0 \sigma_{e}$ \\
\hline Mandel & 20,06 & 5,36 & 2,00 & 1,41 & 1,22 & 1,15 & 1,11 \\
\hline Gráfico 3 & 394,82 & 31,74 & 4,43 & 1,99 & 1,49 & 1,29 & 1,21 \\
\hline Proposta & 653,56 & 145,23 & 17,85 & 2,13 & 1,52 & 1,31 & 1,22 \\
\hline Haworth & 1259,94 & 243,63 & 24,74 & 2,32 & 1,59 & 1,35 & 1,24 \\
\hline
\end{tabular}

Tabela 4. Valores do NMA para alterações no desvio-padrão $\sigma$.

\begin{tabular}{|c|c|c|c|c|c|c|c|c|c|c|c|}
\hline \multirow{2}{*}{ Gráfico } & \multirow{2}{*}{$\frac{\mathrm{NMA}_{0}}{1,0 \sigma}$} & \multicolumn{10}{|c|}{$\mathrm{NMA}_{\mathrm{f}}$} \\
\hline & & $1,2 \sigma$ & $1,4 \sigma$ & $1,6 \sigma$ & $1,8 \sigma$ & $2,0 \sigma$ & $2,2 \sigma$ & $2,4 \sigma$ & $2,6 \sigma$ & $2,8 \sigma$ & $3,0 \sigma$ \\
\hline Mandel & 20,06 & 9,76 & 6,27 & 5,57 & 3,69 & 3,08 & 2,66 & 2,44 & 2,25 & 2,07 & 1,94 \\
\hline Gráfico 3 & 394,82 & 83,90 & 31,58 & 16,08 & 10,53 & 7,40 & 5,70 & 4,66 & 3,99 & 3,49 & 3,17 \\
\hline Proposta & 653,56 & 114,19 & 40,46 & 19,86 & 12,09 & 8,40 & 6,38 & 5,15 & 4,31 & 3,72 & 3,31 \\
\hline Haworth & 1259,94 & 188,88 & 57,82 & 26,46 & 15,02 & 10,14 & 7,50 & 5,86 & 4,89 & 4,15 & 3,64 \\
\hline
\end{tabular}

Tabela 5. Valores do NMA para alterações no coeficiente de inclinação de $x_{1}$.

\begin{tabular}{crrrrrrr}
\hline \multirow{2}{*}{ Gráfico } & \multicolumn{7}{c}{ NMA $_{0}$} \\
\cline { 2 - 7 } & \multicolumn{1}{c}{$x_{1}$} & $x_{1}+0,5 \sigma_{x}$ & $x_{1}+1,0 \sigma_{x}$ & $x_{1}+1,5 \sigma_{x}$ & $x_{1}+2,0 \sigma_{x}$ & $x_{1}+2,5 \sigma_{x}$ & $x_{1}+3,0 \sigma_{x}$ \\
\hline Mandel & 20,06 & 19,26 & 18,52 & 16,71 & 15,40 & 14,62 & 12,95 \\
Gráfico 3 & 394,82 & 370,90 & 345,10 & 283,29 & 266,18 & 224,04 & 185,99 \\
Proposta & 653,56 & 685,49 & 703,58 & 817,19 & 952,33 & 1039,28 & 1269,19 \\
Haworth & 1259,94 & 1270,00 & 1325,49 & 1520,93 & 1763,05 & 1962,33 & 2224,68 \\
\hline
\end{tabular}

Como é possível observar na Tabela 5, o gráfico de controle de Mandel (1969) possui elevada sensibilidade a essas variações, gerando grande taxa de alarmes falsos. 0 gráfico $3 \sigma$ apresentou um resultado satisfatório, mas que foi piorando à medida que aumenta-se o valor da média de $x_{1}$. Os gráficos de controle de regressão proposto no presente trabalho e o de Haworth (1996) apresentaram um desempenho superior, já que foram bastante insensíveis a essas modificações. Esse desempenho superior está associado ao afastamento de $h_{j j}$ do centróide da elipse delimitada pelos valores das variáveis de controle utilizadas na Fase 1, que alargaram os limites de controle.

De forma geral, quando comparado aos outros três gráficos estudados, o gráfico de controle de regressão proposto apresentou bom desempenho em todas as alterações simuladas, sobretudo para alterações superiores a $1,5 \sigma_{e}$ nos coeficientes de regressão e 2,2 do desvio-padrão dos resíduos. 0 gráfico de controle de Mandel (1969) apresentou boa sensibilidade às alterações simuladas, só que esse desempenho é prejudicado pela elevada taxa de alarmes falsos desse gráfico de controle, o que, em uma situação real, geraria muitas intervenções desnecessárias, desestimulando o uso do gráfico por parte tos operadores.

\section{Conclusões}

Para o uso dos gráficos de controle tradicionais é necessário supor que as variáveis monitoradas sejam independentes e identicamente distribuídas. Quando ocorrem frequentes ajustes nas variáveis de controle, a distribuição da característica de qualidade do processo pode ser alterada significativamente, fazendo com que os gráficos de controle clássicos sejam ineficientes. Nessa situação, se existir uma relação linear entre a característica de qualidade e as variáveis de controle, é possível utilizar o gráfico de controle de regressão.

0 presente artigo teve como objetivo principal propor um método para a aplicação dos gráficos de controle de regressão em um sistema produtivo, que foi elaborado a partir de outros métodos encontrados na literatura. Dessa forma, foi proposto um método dividido em duas fases inter-relacionadas: análise retrospectiva (Fase 1) e monitoramento do processo propriamente dito (Fase 11).

A Fase 1 do método proposto inclui as etapas de coleta de dados, estimação do modelo de regressão linear e verificação da estabilidade do processo no período em que a amostra foi coletada. Se o processo for considerado como estando sob controle estatístico, e assumindo que o modelo 
de regressão estimado na Fase 1 do processo esteja correto, prossegue-se à Fase 11 do método proposto, que consiste na coleta de amostras individuais do processo, verificação da extrapolação da região original dos dados e monitoramento da característica de qualidade.

A principal justificativa para o método proposto é facilitar a aplicação dos gráficos de controle de regressão. Seguindo esse princípio, propôs-se também uma modificação no gráfico de controle de regressão múltipla, adaptando-se os limites de controle desse gráfico, de forma a permitir o monitoramento direto da característica de qualidade do processo, facilitando a interpretação do processo por parte dos operários.

Durante a aplicação do método proposto, surgiu a necessidade de criar um procedimento que facilite a verificação da extrapolação do intervalo de investigação das variáveis de controle. Nesse caso, adaptou-se graficamente um procedimento encontrado na literatura para a verificação se o conjunto das variáveis de controle extrapola a região original de dados. Essa adaptação deu origem ao gráfico de controle de extrapolação, que pode ser considerado também uma importante contribuição deste trabalho.

0 método proposto foi aplicado e validado em um processo de extrusão de bandas de rodagem de uma indústria de borrachas. Nessa aplicação, explicitaram-se as etapas que compõem as fases do método proposto, de forma a ressaltar a facilidade que a sistematização do método proposto pode proporcionar. Durante a aplicação, o gráfico de controle de extrapolação indicou um ponto fora de controle e o gráfico de controle de regressão apontou dois pontos como discrepantes.

0 gráfico de controle de regressão proposto apresentou bom desempenho nas simulações estudadas, quando comparado aos demais gráficos de controle, já que apresentou alto $\mathrm{NMA}_{0}$, que reflete baixa ocorrência de alarmes falsos, e boa sensibilidade às alterações nos coeficientes de regressão e do desvio-padrão, especialmente para as situações em que ocorrem alterações superiores a 1,5 desvios-padrão nesses parâmetros.

Para trabalhos futuros, sugere-se a proposta de um método para a aplicação do gráfico de controle de regressão não linear, semelhante ao método proposto no presente artigo.

\section{Referências}

AIZENCHTADT, E.; INGMAN, D.; FRIEDLER, E. Quality control of wastewater treatment: a new approach. European Journal of Operations Research, v. 189, p. 445-458, 2008.
FAlTIN, F. W. et al. Considerations in the monitoring of the autocorrelated and independent data. Journal of Quality Technology, v. 29, n. 2, p. 131-133, 1997.

HAUCK, D. J.; RUNER, G. C.; MONTGOMERY, D. C. Multivariate statistical process monitoring and diagnosis with grouped regression-adjusted variables. Comunications in Statistics Simulation and Computation, v. 28, n. 2, p. 309-328, 1999.

HAWKINS, D. M. Multivariate quality control based on reegression-adjusted variables. Technometrics, v. 33, n. 1, p. 61-75, 1991.

HAWKINS, D. M. Regression adjustment for variables in multivariate quality control. Journal of Quality Technology, v. 25, n. 3, p. 170-182, 1993.

HAWORTH, D. A. Regression control chart to manage software maintenance. Journal of Software Maintenance, v. 8, n. 1, p. 35-48, 1996.

JACOBI, L. F.; SOUZA, A. M.; PEREIRA, J. E. S. Gráfico de controle de regressão aplicado na monitoração de processos. Revista Produção, v. 12, n. 1, p. 46-59, 2002.

JEARKPAPORN, D. et al. Process monitoring for correlated gamma-distributed data using generalized-linear-modelbased control charts. Quality and Reliability Engineering International, v. 19, n. 6, p. 477-491, 2003.

JEARKPAPORN, D. et al. Process monitoring for mean shifts for multiple stage processes. International Journal of Production Research, v. 45, n. 3, p. 5547-5570, 2007.

JOHNSON, B.; JOHNSON, C.; SEIBER, J. The use of regression equations for quality control in a pesticide physical property database. Environmental Management, v. 19, n. 1, p. 127-134, 1995.

KANG, L.; ALBIN, S. On-line monitoring when the process yelds linear profiles. Journal of Quality Technology, v. 32, n. 4, p. 418-426, 2000.

LOREDO, E. N.; JEARKPAPORN, D.; BORROR, C. M. Modelbased control chart for autoregressive and correlated data. Quality and Reliability Engineering International, v. 18, n. 6, p. 489-496, 2002.

MANDEL, B. J. The regression control chart. Journal of Quality Technology, v. 1, n. 1, p. 1-9, 1969.

MONTGOMERY, D. C. Introdução ao Controle Estatístico da Qualidade. 4. ed. Rio de Janeiro: Editora LTC, 2004. 513 p.

MONTGOMERY, D. C.; MASTRANGELO, C. M. Some statistical process control methods for autocorrelated data. Journal of Quality Technology, v. 23, n. 3, p. 179-193, 1991.

MONTGOMERY, D. C.; VINING, G. G.; PECK, E. A. Introduction to Linear Regression Analysis. 3 ed. New York: John Wiley \& Sons, 2001. $641 \mathrm{p}$.

NETER, J. et al. Applied Linear Statistical Models. 5 ed. New York: Mc Graw-Hill/Irwin, 2005. 1396 p.

OLIN, B. D. Regression control charts revisited: methodology and cases studies. In: ANNUAL FALL TECHNICAL CONFERENCE - AFTC, 42., 1998, New York. Proceedings... New York: American Society for Quality, 1998. $17 \mathrm{p}$.

OMURA, A. P.; STEFFE, J. H. Mixer viscometry to characterize fluid foods with large particulates. Journal of Food Process Engineering, v. 26, n. 3, p. 435-445, 2003.

SHU, L; TSUNG, F; TSUI, K. L. Run-length perfomance of regression control charts with estimated parameters. Journal of Quality Technology, v. 36, n. 3, p. 280-292, 2004.

SKINNER, K. R.; MONTGOMERY, D. C.; RUNGER, G. C. Process monitoring for multiple count data using generalized linear model-based control charts. International Journal of Production Research, v. 41, n. 6, p. 1167-1180, 2003.

VINING, G. Technical Advice: Phase 1 and phase 11 control charts. Quality Engineering, v. 21, n. 4, p. 478-479, 2009.

WADE, M. R.; WOODAL, W. H. A review and analysis of causeselecting control charts. Journal of Quality Technology, v. 25, n. 3, p. 161-169, 1993. 
WOODALL, W. H. Controversions and contradictions in statistical process control. Journal of Quality Technology, v. 32, n. 4, p. 341-350, 2000.

WOODALL, W. H. Current research on profile monitoring. Revista Produção, v. 17, n. 3, p. 420-425, 2007.

WOODALL, W. H. et al. Using control charts to monitor process and product quality profiles. Journal of Quality Technology, v. 36, n. 3, p. 309-320, 2004.

WOODALL, W. H.; MONTGOMERY, D.C. Research issues and ideas in statistical process control. Journal of Quality Technology, v. 31, n. 4, p. 376-386, 1999.
ZHANG, Z. X. Cause-selecting control charts - a new type of quality control charts. The QR Journal, v. 12, p. 221-225, 1985.

\section{Agradecimentos}

Gostaríamos de agradecer aos editores e revisores anônimos pelas valiosas sugestões e à CAPES pelo apoio financeiro na execução deste trabalho. Também agradecemos a Ângelo Sant'ana, Ivan Allaman, Rodrigo Coster e Walmes Zeviani pelas dicas sobre programação em R.

\section{Method for applying regression control charts to process monitoring}

\section{Abstract}

This work proposes a method for the application of regression control charts in the monitoring of manufacturing processes. The proposed method is presented in two phases: retrospective analysis (Phase 1) and process monitoring (Phase 11). It includes a simple modification of the multiple regression control chart, allowing the monitoring of the values of quality characteristics of the process, instead of monitoring the regression standardized residuals. It also proposes an extrapolation control chart, which verifies whether the control variables extrapolate the set of data used in regression model estimation. The proposed method was successfully applied in a rubber manufacturing process. The Average Run length (ARL) distribution was estimated using the Monte Carlo method, proving the efficiency of the proposed chart in detecting some alterations in process parameters.

\section{Keywords}

Regression control chart. Linear regression models. Average run length.

Apêndice 1. Modificação no Gráfico de Controle de Regressão.

Primeiramente, para o gráfico de controle proposto por Haworth (1996), adota-se $L=3$ ao invés de utilizar $t_{\alpha / 2, n-p}$. Assim, os limites de controle e linha central são:

$$
L I C_{j}=-3 \quad L C=0 \quad L S C_{j}=+3
$$

A variável monitorada pelo gráfico de controle são os resíduos na forma Student:

$$
\text { Variável monitorada }=\frac{e_{j}}{\hat{\sigma} \sqrt{1+h_{j j}}}
$$

A seguir, multiplicam-se os resíduos padronizados, os limites de controle e a linha central pelo desvio-padrão da previsão de uma nova observação. Os novos limites de controle e a linha central passam a ser:

$$
L I C_{j}=-3 \hat{\sigma} \sqrt{1+h_{j j}} \quad L C=0 \quad L S C_{j}=+3 \hat{\sigma} \sqrt{1+h_{j j}}
$$

Após essa modificação inicial, a variável a ser monitorada passa a ser o resíduo do modelo $\left(e_{j}\right)$ :

$$
\text { Variável monitorada }=\frac{e_{j}}{\hat{\sigma} \sqrt{1+h_{j j}}} \times \hat{\sigma} \sqrt{1+h_{j j}}=e_{j}
$$

A seguir, soma-se o valor previsto pelo modelo à variável monitorada aos limites de controle e à linha central. Dessa forma, temos:

$$
L I C_{j}=\hat{y}_{i}-3 \hat{\sigma} \sqrt{1+h_{j j}} \quad L C=\hat{y}_{i} \quad L S C_{j}=\hat{y}_{i}+3 \hat{\sigma} \sqrt{1+h_{j j}}
$$

A variável monitorada passa a ser o valor observado da característica de qualidade, já que:

$$
\text { Variável monitorada }=e_{j}+\hat{y}_{j}=\left(y_{j}-\hat{y}_{j}\right)+\hat{y}_{j}=y_{j}
$$

